\title{
A TRANSFER THEOREM FOR NONSTANDARD UNISERIALS
}

\author{
PAUL C. EKLOF
}

(Communicated by Louis J. Ratliff, Jr.)

\begin{abstract}
A general theorem is described and proved which allows the transfer of results about the existence of nonstandard uniserial modules over a valuation domain from models of $\mathrm{ZFC}+\diamond$ to all models of ZFC.
\end{abstract}

An $R$-module $M$ is called uniserial if its submodules form a chain under inclusion. A ring $R$ is called a valuation ring if it is a commutative ring with 1 , which is uniserial as a module over itself; $R$ is a valuation domain if it is also an integral domain. If $R$ is a valuation domain, the canonical examples of uniserial $R$-modules are those of the form $J / I$ where $I \subseteq J$ are $R$-submodules of $Q$, the quotient field of $R$; these are the standard uniserial $R$-modules.

For a long time it was unknown whether there were nonstandard uniserial modules. Their existence was first established by Shelah [Sh2], who forced to obtain a particular model of ZFC (ordinary, Zermelo-Frankel, set theory with the axiom of choice) containing a model of arithmetic with appropriate properties that allowed one to construct a divisible nonstandard uniserial module; he then used a model-theoretic absoluteness argument (based on the completeness theorem for stationary logic) to show that a suitable model of arithmetic existed in any model of ZFC, and hence that it was a theorem of ZFC that there were divisible nonstandard uniserial modules.

Immediately afterwards, Fuchs and Salce [FS] constructed a divisible nonstandard uniserial module using $\diamond$ (a combinatorial principle that is consistent with, but independent of, ZFC). In [FSh], this construction plus a different model-theoretic absoluteness argument (based on [Sh1]) is used to show that it is a theorem of ZFC that there are divisible nonstandard uniserials.

Recently a great deal of work has been done, notably by Bazzoni and Salce, on properties which differentiate nonstandard uniserials. In [BS1] are defined six different classes of nonstandard uniserial modules, distinguished by which homomorphic images of the module are nonstandard. On the one extreme are the strongly nonstandard ones for which all nonzero quotients are nonstandard. (These actually form two classes, depending on whether the modules are divisible or bounded.) At the other extreme are the barely nonstandard ones for

Received by the editors July 2, 1990 and, in revised form, October 16, 1990.

1980 Mathematics Subject Classification (1985 Revision). Primary 13A18, 13L05; Secondary 03C80, 03E35.

Key words and phrases. Valuation domain, uniserial, nonstandard uniserial, many-sorted language, transfer theorem. 
which all proper quotients are standard. Bazzoni and Salce prove (using $\diamond$ ) that it is consistent with ZFC that each of these classes is nonempty (for some valuation domain).

It is a natural question-in fact, posed by Salce to the author-whether it is provable in ZFC that these six classes are nonempty. Bazzoni and Salce have done other work which raises similar questions (cf. [BS2] and [BS3]). Thus it seems desirable to have a general tool that would allow one to transfer results about the existence of nonstandard uniserials proved using $\diamond$ to the same results proved in ZFC. The purpose of this note is to observe that such a result can be derived from the method of proof used in the Fuchs-Shelah paper:

Transfer Theorem. Let $\varphi_{0}, \ldots, \varphi_{m}$ be sentences of the first-order two-sorted language of $R$-modules such that it is provable from ZFC $+\diamond$ that there is a valuation domain $R$ and nonstandard uniserial $R$-modules $U_{0}, \ldots, U_{m}$ so that $\varphi_{i}$ is true in $U_{i}$. Then it is provable from ZFC that there is a valuation domain $R^{\prime}$ and nonstandard uniserial $R^{\prime}$-modules $U_{0}^{\prime}, \ldots, U_{m}^{\prime}$ so that $\varphi_{i}$ is true in $U_{i}^{\prime}$.

The two-sorted language referred to in the statement of the theorem is a natural language in which to speak about modules over a ring and one powerful enough to express many, if not all, of the properties of uniserial modules which have been studied. The first section is devoted to explaining this language and its applications-hopefully in such a way that the Transfer Theorem can be employed by an algebraist not versed in logic. Among the consequences of the Transfer Theorem discussed in the first section, is the following weak version which is sufficient for some uses. (For the notation, see $\S 1$.)

Corollary 1. Let $\varphi_{0}, \ldots, \varphi_{m}$ be Boolean combinations of statements of the form $X \subseteq Y$, where $X$ and $Y$ are $0, U, U^{c}, U_{c}$, or $U[P]$ (subsets of $U$ ) or $X$ and $Y$ are $0, R, P, U^{\#}, U_{\#}, \operatorname{Ann}(U), \operatorname{Ann}\left(U / U^{c}\right)$ or $\operatorname{Ann}\left(U / U_{c}\right)$ (subsets of $R)$. Suppose that it is provable from $\mathrm{ZFC}+\diamond$ that there is a valuation domain $R$ and nonstandard uniserial $R$-modules $U_{0}, \ldots, U_{m}$ so that $\varphi_{i}$ is true in $U_{i}$. Then it is provable from ZFC that there is a valuation domain $R^{\prime}$ and nonstandard uniserial $R^{\prime}$-modules $U_{0}^{\prime}, \ldots, U_{m}^{\prime}$ so that $\varphi_{i}$ is true in $U_{i}^{\prime}$.

Here, a Boolean combination of statements means a conjunction of disjunctions of the statements or their negations. Using Corollary 1 we derive in $\S 1$ the following, answering Salce's question.

Corollary 2. It is provable in ZFC that there exist nonstandard uniserials in each of the six classes described in [BS1].

A uniserial module is said to have principal annihilators if the annihilator of one (hence every) nonzero element is a principal ideal. This is not expressible by a Boolean combination of statements as in Corollary 1 , but we show in $\S 1$ that it is expressible by a sentence of the two-sorted language. Thus, as another application of the Transfer Theorem we get the following.

Corollary 3. If it is provable from $\mathrm{ZFC}+\diamond$ that there exists a nonstandard uniserial module with principal annihilators (respectively, without principal annihilators) satisfying a Boolean combination $\varphi$ of statements as in Corollary 1 , then it is provable in ZFC that there is a nonstandard uniserial satisfying 
$\varphi$ which has principal annihilators (respectively, which does not have principal annihilators).

By applying Corollary 3 to a consistency result from [BS2] we get another result about the existence of a certain type of nonstandard uniserial:

Corollary 4. It is provable in ZFC that there exists a (barely nonstandard) uniserial of type $J / A$ where $A$ is archimedean, not principal, and not isomorphic to $P$.

The Transfer Theorem and Corollaries 1 and 3 also apply when ZFC $+\diamond$ is placed by many other axiomatic extensions of ZFC; see Remark 1 in $\S 2$.

The first section does not presume a knowledge of [FSh]. The second section, which proves the Transfer Theorem, does assume familiarity with [FSh]; it also discusses some strengthenings of the Transfer Theorem.

\section{The TWO-SORTED LANGUAGE}

To begin, let $R$ be an arbitrary ring with 1 . We will describe a precise symbolic language in which to express properties of $R$-modules, including properties of the ring $R$. (See [Ba, p. 42] or [Fe] for more on many-sorted languages.) We will use two sorts of variables, one for elements of the ring and one for elements of the module. Elements of the ring will always be denoted by lower case Greek letters: $\rho, \sigma, \tau, \ldots$ (We will reserve the letters $\psi$ and $\varphi$ for formulas.) Elements of the module will be denoted by lower case Latin letters: $u, v, w, \ldots$. We will have three constant symbols: 0 for the zero element of the module; $\theta$ for the zero element of the ring; and 1 for the multiplicative identity of the ring. The basic building blocks, the atomic formulas, will be those of the form

$$
f\left(1, \rho_{1}, \ldots, \rho_{n}\right)=\theta
$$

where $f\left(X_{0}, X_{1}, \ldots, X_{n}\right)$ is a polynomial with coefficients in $\mathbb{Z}$, or of the form

$$
\sum_{i \leq n} t_{i} u_{i}=0
$$

where each $t_{i}$ is of the form $f_{i}\left(1, \rho_{1}, \ldots, \rho_{n}\right)$ where $f_{i}$ is a polynomial as above.

The formulas of our language are those obtained from the atomic formulas by a finite number of applications of the following rule: if $\varphi$ and $\psi$ are formulas, then so are $(\varphi \wedge \psi),(\varphi \vee \psi),(\varphi \rightarrow \psi),(\neg \varphi), \forall \rho \varphi, \forall u \varphi, \exists \rho \varphi$, and $\exists u \varphi$. (Here $\rho$ stands for any variable of the ring sort and $u$ for any variable of the module sort.) An occurrence of a variable $\rho$ (respectively, $u$ ) in a formula $\varphi$ is called free if it is not within the scope of a quantifier $\forall \rho$ or $\exists \rho$ (respectively, $\forall u$ or $\exists u$ ). A formula without any free occurrences of variables is called a sentence.

Given a ring $R$ and an $R$-module $U$, there is a natural sense in which a given sentence $\varphi$ expresses properties of $U$ and/or $R$ (which may or may not be true). We write ${ }_{R} U \models \varphi$ or just $U \models \varphi$ to mean that $\varphi$ is true in the $R$-module $U$. (In this case, we also say $U$ satisfies $\varphi$.) Rather than give the formal definition, we will illustrate it with some examples. Notice first that whether or not ${ }_{R} U$ satisfies $\varphi$ may depend (wholly) on properties of the ring $R$. For example, if $\varphi$ is the sentence $\forall \rho \forall \sigma(\rho \sigma=\sigma \rho))$, then ${ }_{R} U \models \varphi$ if and 
only if $R$ is a commutative ring. (In this case, $\varphi$ is true in one $R$-module $U$ if and only if it is true in all $R$-modules if and only if $R$ is commutative.)

If $\varphi_{1}$ is the sentence $\forall u \forall v \exists \tau(\tau u=v \vee \tau v=u)$, then $U \models \varphi_{1}$ if and only if $U$ is a uniserial module. If $\varphi_{2}$ is the sentence

$$
\forall \rho \forall \sigma(\rho \sigma=\theta \rightarrow(\rho=\theta \vee \sigma=\theta)) \wedge \forall u \forall \rho(\rho \neq \theta \rightarrow(\exists v \rho v=u)),
$$

then $U \models \varphi_{2}$ if and only if $R$ has no zero-divisors and $U$ is a divisible $R$ module. $(\rho \neq \theta$ is an abbreviation for $\neg(\rho=\theta)$.)

For definiteness, we will assume from now on that $R$ is a valuation domain.

An $R$-module $U$ is a torsion module if and only if it satisfies the sentence

$$
\forall v \exists \sigma(\sigma \neq \theta \wedge \sigma v=0) .
$$

If $\psi$ is a formula with one variable which occurs free, then there is a natural sense in which, in a given $R$-module $U, \psi$ defines a subset of $R$ (if the free variable is of the ring sort) or of $U$ (if the free variable is of the module sort). Again, we will illustrate with some examples. (The free occurrences of the variable are underlined.) If $\psi$ is the formula $\neg \exists \sigma(\sigma \rho=1)$, then $\psi$ defines the maximal ideal $P$ in $R$. If $\psi_{1}$ is the formula $\exists u \forall \bar{v}(\neg(\underline{\rho} v=u))$, then, for a given $R$-module $U, \psi_{1}$ defines the prime ideal

$$
U^{\#}=\{r \in R: r U<U\}
$$

of $R$ associated with $U$ (cf. [FS, p. 34]). Similarly, the formula $\psi_{2}$ :

$$
\exists u(u \neq 0 \wedge \underline{\rho} u=0)
$$

defines the prime ideal $U_{\#}=\{r \in R: r u=0$ for some $0 \neq u \in U\}$. Thus $U$ is torsion-free if and only if $U$ satisfies the sentence

$$
\forall \rho\left(\psi_{2}(\rho) \rightarrow \rho=\theta\right) \text {. }
$$

The annihilator of $U, \operatorname{Ann}(U)$, is defined by the formula $\psi_{3}$ :

$$
\forall u(\underline{\rho} u=0) \text {. }
$$

We can use these formulas to define others. Thus if $\psi_{4}$ is the formula

$$
\forall \rho\left(\psi_{1}(\rho) \rightarrow \rho \underline{u}=0\right)
$$

then $\psi_{4}$ defines the upper threshold submodule

$$
U^{c}=\left\{a \in U: r a=0 \text { for all } r \in U^{\#}\right\}
$$

of $U$ (cf. [BS1, p. 297]). Similarly, but using a more informal notation, the formula $\psi_{5}, \exists \rho\left(\neg\left(\rho \in U^{\#}\right) \wedge \rho \underline{u}=0\right)$ defines the lower threshold submodule $U_{c}$. Moreover, $\operatorname{Ann}\left(U / U^{c}\right)$ is defined by $\forall u \psi_{4}(\rho u)$.

Using these formulas we can easily express statements of the form $X \subseteq$ $Y$-as in Corollary 1 -in the two-sorted language. For example, $U^{c} \subseteq U_{c}$ is expressed by the sentence $\forall u\left(\psi_{4}(u) \rightarrow \psi_{5}(u)\right)$. Also, $U_{\#} \subseteq U^{\#}$ is expressed by the sentence $\forall \rho\left(\psi_{2}(\rho) \rightarrow \psi_{1}(\rho)\right)$. Then Corollary 1 follows easily from the Transfer Theorem. Moreover, Corollary 2 follows from Corollary 1 since the six classes of [BS1] can be defined in terms of such Boolean combinations (cf. [BS2]). For example, the class of strongly nonstandard bounded uniserials $\left(\mathscr{U}_{2}\right.$ of [BS2]) is defined (among nonstandard uniserials) by

$$
\neg\left(U_{\#} \subseteq U^{\#}\right) \wedge \neg\left(U^{\#} \subseteq \operatorname{Ann}(U)\right) \wedge \neg(\operatorname{Ann}(U) \subseteq 0) .
$$


Note that we cannot say in our two-sorted language that $U$ is a nonstandard uniserial. Indeed for any sentence $\varphi$ of the language, if $\varphi$ is true in some uniserial module, then by the downward Löwenheim-Skolem theorem, there is a countable uniserial $U$ (with countable $R$ ) such that $\varphi$ is true in $U$ (cf. [ $\mathrm{Ba}$, p. 10]). But then $U$ is a standard uniserial by [FS, p. 142].

We conclude this section by discussing some other properties of uniserial modules which arise in [BS2] and [BS3] and which involve the nature of the type, $J / A$, of $U$. ( $U$ is said to have type $J / A$ if there exists $0 \neq u \in U$, such that $\operatorname{Ann}(u)=A$ and $u$ is divisible by precisely the elements $r$, such that $r^{-1} \in J$.) Here we must take a more indirect approach. (See also Remark 3 of §2.) To say that $U$ has type $J / R$ for some $J$ means that the annihilator of each nonzero element of $U$ is principal; that is, $U$ satisfies the sentence $\varphi_{3}: \forall u \exists \rho \forall \sigma(\sigma u=0 \longleftrightarrow(\exists \tau \tau \rho=\sigma))$. Then $U$ does not have principal annihilators if and only if $U \models \neg \varphi_{3}$. Corollary 3 follows easily from the Transfer Theorem.

To say that $U$ is of type $J / A$ where $A$ is archimedean (i.e. $A^{\#}=P$ ) means that $U$ satisfies the sentence $\forall \rho\left(\psi(\rho) \rightarrow \psi_{2}(\rho)\right)$ We can also express this by saying $U_{\#}=P$. To say that $U$ is of type $J / A$ where $A$ is isomorphic to $P$ means that $U$ satisfies the sentence

$$
\exists u \exists \rho \forall \sigma(\sigma u=0 \longleftrightarrow \exists \tau(\tau \in P \wedge \sigma=\rho \tau)) .
$$

Now Corollary 4 follows directly from Corollary 3 (or from the Transfer Theorem) and the fact, proved in [BS2], that it is a consequence of ZFC $+\diamond$ that there exists a barely nonstandard uniserial module $U$ which does not have principal annihilators and satisfies $U_{\#}=P$.

\section{Proof of the Transfer Theorem}

We begin with the case $m=0$ of the theorem. As in [FSh], we consider a class of multisorted models, but since we do not want to confine ourselves to divisible torsion uniserials, we need a more complicated class. Let $\mathscr{K}^{\prime}$ be the class of multisorted models

$$
N=\left(L^{N}, R^{N}, Q^{N}, U^{N}, U_{\#}^{N}, R_{\#}^{N}, A^{N}, J^{N}, T^{N}, f^{N}, g^{N}\right)
$$

where $L^{N}, R^{N}, Q^{N}$ are as in [FSh, p. 27], $U^{N}$ is a uniserial $R^{N}$-module, $U_{\#}^{N}$ is the prime ideal defined by $\psi_{2}$, and $R_{\sharp}^{N} \subseteq Q^{N}$ is $R_{U_{\sharp}^{N}}^{N}$, i.e. $R^{N}$ localized at $U_{\#}^{N}$. We require also that $\left(U^{N}\right)^{\#} \subseteq U_{\#}^{N}$, so that $U^{N}$ is canonically an $R_{\#}^{N}$ module; in what follows we will view it as such. (We do not want to assume that $R^{N}=R_{\#}^{N}$ since this property is expressible in the two-sorted language.) In addition:

(a) $J^{N}$ is an $R_{\#}^{N}$-submodule of $Q^{N}$ containing $R_{\#}^{N} ; A^{N}$ is an ideal of $R_{\#}^{N}$, or $A^{N}=R_{\#}^{N}$;

(b) $f^{N}: L^{N} \rightarrow R_{\#}^{N} \backslash\{0\}$ such that:

( $\alpha) s<t$ in $L^{N}$ implies that $f^{N}(s)$ divides $f^{N}(t)$ in $R_{\#}^{N}$; and

$\left(\beta^{\prime}\right)$ for all $r \in R_{\#}^{N}, r^{-1} \in J^{N}$ if and only if there exists $s \in L^{N}$ such that $r$ divides $f^{N}(s)$;

(c) $g^{N}: L^{N} \rightarrow U^{N}$ such that:

$(\gamma) s<t$ in $L^{N}$ implies that $g^{N}(s) R_{\#}^{N} \subseteq g^{N}(t) R_{\#}^{N}$ in $U^{N}$;

$\left(\delta^{\prime}\right)$ for each $s \in L^{N}, \operatorname{Ann}\left(g^{N}(s)\right)=f^{N}(s) A^{N}$; 
( $\epsilon)$ for all $u \in U^{N}$, there is $s \in L^{N}$ such that $u \in g^{N}(s) R_{\#}^{N}$;

(d) $T^{N}$ is a tree, as in [FSh, p. 28] except that $T_{t}^{N}$ is defined to be the set of all isomorphisms

$$
\phi_{t}: f^{N}(t)^{-1} R_{\#}^{N} / A^{N} \rightarrow g^{N}(t) R_{\#}^{N} .
$$

More precisely this means: the element $\phi_{t}$ of $T_{t}^{N}$ is identified with the unit $c_{t}$ of $R_{\#}^{N}$ so that $\phi_{t}\left(f^{N}(t)^{-1}\right)=g^{N}(t) c_{t}$. (This gives an isomorphism by $\left(\delta^{\prime}\right)$.) If $s \leq t$, then $\phi_{s}$ is defined to be $\leq \phi_{t}$ if and only if $g^{N}(s) c_{s}=$ $\left(f^{N}(t) f^{N}(s)^{-1}\right) g^{N}(t) c_{t}$ (i.e., $\phi_{s}$ is the restriction of $\left.\phi_{t}\right)$.

With this understanding, one can easily check that $\mathscr{K}^{\prime}$ is the class of models of a first order theory $\Gamma^{\prime}$. Then, the key lemma is:

Lemma. For $N \in \mathscr{K}^{\prime}, U^{N}$ is standard if and only if $T^{N}$ has a full branch.

Proof. Note that $U^{N}$ is standard as $R^{N}$-module if and only if it is standard as $R_{\#}^{N}$-module. If $U^{N}$ is standard, then since $U^{N}$ has type $J^{N} / A^{N}$, there is an isomorphism

$$
\phi: J^{N} / A^{N} \rightarrow U^{N}
$$

For each $t \in L^{N}$ the image of $f^{N}(t)^{-1} R_{\#}^{N} / A^{N}$ under $\phi$ must be $g^{N}(t) R_{\#}^{N}$ (by $\left(\delta^{\prime}\right)$ and using the fact that $\left(U^{N}\right)_{\#} R_{\#}^{N}=$ the maximal ideal of $\left.R_{\#}^{N}=\left(A^{N}\right)^{\#}\right)$. If for each $t \in L^{N}$ we let $\phi_{t}$ be $\phi\left\lceil f^{N}(t)^{-1} R_{\#}^{N} / A^{N}\right.$, then $\left\{\phi_{t}: t \in L^{N}\right\}$ is a full branch of $T^{N}$.

Conversely, if $B$ is a full branch of $T^{N}$, then

$$
\bigcup\left\{\phi_{t}:\left\{\phi_{t}\right\}=B \cap T_{t}^{N}\right\}
$$

is an isomorphism of $J^{N} / A^{N}$ with $U^{N}$.

Now suppose that we are given a sentence, $\varphi_{0}$, of the two-sorted language of modules. Then $\varphi_{0}$ may be translated directly into a sentence, $\tilde{\varphi}_{0}$, of the language of the models in $\mathscr{K}^{\prime}$ so that a member $N$ of $\mathscr{K}^{\prime}$ satisfies $\tilde{\varphi}_{0}$ if and only if the $R^{N}$-module $U^{N}$ satisfies $\varphi_{0}$. Suppose that it is consistent that there is a nonstandard uniserial $R$-module $U$ in which $\varphi_{0}$ is true. Regard $U$ as an $R_{U_{*}}$-module. By [BS1, Lemma 1.2] or [FS, Lemma VII.1.2], $U$ is, without loss of generality, the direct limit of submodules $r_{\sigma}^{-1} R_{U_{*}} / A(\sigma<\kappa)$ of $Q$ where the connecting homomorphisms $\eta_{\sigma}^{\tau}: r_{\sigma}^{-1} R_{U_{*}} / A \rightarrow r_{\tau}^{-1} R_{U_{*}} / A \quad(\sigma<\tau<\kappa)$ are given by multiplications by units of $R_{U_{*}}$. We let $L^{N}$ be $\kappa, R^{N}$ be $R, U^{N}$ be $U, R_{\#}^{N}$ be $R_{U_{\#}}, A^{N}$ be $A, J^{N}$ be $\bigcup_{\sigma<\kappa} r_{\sigma}^{-1} R_{U_{*}}$, and for all $\sigma \in L^{N}$, define $f^{N}(\sigma)$ to be $r_{\sigma}$ and $g^{N}(\sigma)$ to be the canonical image of $r_{\sigma}^{-1}+A$ in the direct limit. In this way we obtain a model $N$ of $\Gamma^{\prime} \cup\left\{\tilde{\varphi}_{0}\right\}$, which does not have a full branch by the lemma, since $U$ is nonstandard.

The proof is now finished as in [FSh]. Let $\Psi$ be

$\left\{\neg(\exists \bar{y}) \psi_{\phi}(\bar{y}): \phi(x, \bar{y})\right.$ is a first-order formula in the language of $\left.\mathscr{K}^{\prime}\right\}$.

Thus $\Psi$ says that no first-order formula defines a full branch of $T^{N}$. By hypothesis and the lemma, it is true in an extension, $V^{\prime}$, of the universe, $V$, (namely a generic extension in which $\diamond$ is true) that

$$
\Gamma^{+} \stackrel{\text { def }}{=} \Gamma^{\prime} \cup\left\{\tilde{\varphi}_{0}\right\} \cup \Psi
$$

has a model. Thus there is no proof from $\Gamma^{+}$of an inconsistency in $V^{\prime}$ and hence none in $V$-since $V^{\prime} \supseteq V$. Then by Gödel's completeness theorem and 
Theorem 12 of [Sh1], it is provable in ZFC that $\Gamma^{+}$has a model $N^{\prime}$ such that $T^{N^{\prime}}$ has no full branches (except for definable ones, which are excluded by the definition of $\Psi$ ). If we let $R^{\prime}=R^{N^{\prime}}$ and $U_{0}^{\prime}=U^{N^{\prime}}$, then we have obtained in ZFC a nonstandard uniserial in which $\varphi_{0}$ is true.

For the general case $m>0$, we need only to consider models of the form

$$
N=\left(L_{j}^{N}, R^{N}, Q^{N}, U_{j}^{N}, U_{\#, j}^{N}, R_{\#, j}^{N}, A_{j}^{N}, J_{j}^{N}, T_{j}^{N}, f_{j}^{N}, g_{j}^{N}: j \leq m\right)
$$

and sentences $\tilde{\varphi}_{j}$ in the language of these models which say that the $R^{N}$-module $U_{j}^{N}$ satisfies $\varphi_{j}$.

Remarks. (1) By the same kind of argument, one can replace in the Transfer Theorem "provable from ZFC $+\diamond$," by "provable from $\mathrm{ZFC}+\mathrm{V}=\mathrm{L}$ " or "provable in a forcing extension of the universe."

(2) By similar means one can show that if it is consistent with ZFC that there are $k$ different (nonisomorphic) nonstandard $R$-uniserials satisfying a given $\varphi$, then it is provable in ZFC that there is a valuation domain $R^{\prime}$ such that there are $k$ different (nonisomorphic) nonstandard $R^{\prime}$-uniserials satisfying $\varphi$. One can also require $U_{j}^{\prime}$ and $R^{\prime}$ to be of a given uncountable cardinality $\lambda$. (For $\lambda$ the successor of a regular cardinal, this follows from [Sh1]; otherwise, use [MSh].)

(3) In order to speak about the type, $J / A$ of a uniserial module in a natural way (rather than indirectly as we have done in Corollaries 3 and 4), one could add to the two-sorted language unary predicates $J$ and $A$ and prove, completely analogously, a Transfer Theorem for this language.

(4) The Transfer Theorem is used in [BS3] to show that several new classes of nonstandard uniserials are nonempty in ZFC. There is, however, one class studied there to which the Transfer Theorem does not seem to apply: the class of $U \in \mathscr{U}_{4}$ of type $J / A$ such that $A$ is principal, $P=R p$ is principal and $J^{\#}=\bigcap_{n=1}^{\infty} R p^{n}$ (cf. Proposition 2.1(b)). The problem is in expressing the last equality. In this case, one can use Theorem 6 of [Sh1] to derive a suitable transfer result for uniserials of cardinality $\aleph_{1}$.

Added in revision. Barbara Osofsky has recently given the first direct construction in ZFC of a nonstandard uniserial; her method also constructs nonstandard uniserials of different kinds.

\section{REFERENCES}

[Ba] J. Barwise, An introduction to first-order logic, Handbook of Mathematical Logic, NorthHolland, Amsterdam, 1977, pp. 5-46.

[BS1] S. Bazzoni and L. Salce, On nonstandard uniserial modules over valuation domains and their quotients, J. Algebra 128 (1990), 292-305.

[BS2] _ Elongations of uniserial modules over valuation domains, J. Algebra (to appear).

[BS3] __ Equimorphism classes of uniserial modules over valuation domains, preprint.

[Fe] Applications of many-sorted interpolation theorems, Proceedings of the Tarski Symposium, Amer. Math. Soc., Providence, RI, 1974, pp. 205-224.

[FS] L. Fuchs and L. Salce, Modules over valuation domains, Marcel Dekker, New York, 1985.

[FSh] L. Fuchs and S. Shelah. Kaplansky's problem on valuation rings, Proc. Amer. Math. Soc. 105 (1989), 25-30.

[MSh] A. Mekler and S. Shelah, Compactness for isomorphism quantifiers, preprint. 
[Sh1] S. Shelah, Models with second-order properties. II, Trees with no undefined branches, Ann. of Math. 14 (1978), 73-87.

[Sh2] _ Nonstandard uniserial module over a uniserial domain exists, Around Classification Theory of Models, Lecture Notes in Math., vol. 1182, Springer-Verlag, Berlin and New York (1986), 135-150.

Department of Mathematics, University of California at Irvine, Irvine, California 92717

E-mail address: pceklof@uci.edu 